\title{
Hexagonal Boron Nitride Nanosheets Grown via \\ Chemical Vapor Deposition for Silver Protection
}

\author{
Rui Han ${ }^{\dagger}+$, Majharul H. Khan ${ }^{\S}$, Alexander Angeloski ${ }^{\ddagger}$, Gilberto Casillas",

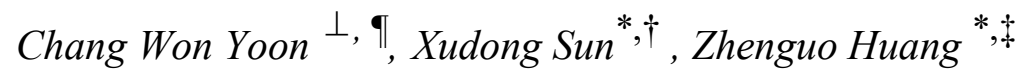

${ }^{\dagger}$ Key Laboratory for Anisotropy and Texture of Materials (Ministry of Education), Northeastern

University, Shenyang 110819, China.

${ }^{\ddagger}$ School of Civil \& Environmental Engineering, University of Technology Sydney, Ultimo,

NSW 2007, Australia.

${ }^{\S}$ Institute for Superconducting and Electronic Materials, University of Wollongong, Wollongong, NSW 2500, Australia.

"Electron Microscopy Centre, University of Wollongong, NSW 2500, Australia. 
${ }^{\perp}$ Center for Hydrogen and Fuel Cell Research, Korea Institute of Science and Technology, Seoul 02792, Republic of Korea.

"KHU-KIST Department of Converging Science and Technology, Kyung Hee University, Seoul 02447, Republic of Korea.

\title{
KEYWORDS
}

2D materials; hexagonal boron nitride; nanosheets; chemical vapor deposition; silver anticorrosion; protective coatings

\begin{abstract}
In this study, hexagonal boron nitride nanosheets (h-BNNS) have been grown on polycrystalline silver substrates via chemical vapor deposition (CVD) using ammonia borane as a precursor. The h-BNNS are of few-atomic-layer thickness and form continuous coverage over the whole Ag substrate. The atomically thin coating poses negligible interference to the reflectivity in the UVvisible range. The nanosheet coating also proves very effective in protecting Ag foil chemically. In contrast to bare Ag foil, the coated one displayed only minor decolorization under high
\end{abstract}


concentration of $\mathrm{H}_{2} \mathrm{~S}$. The study indicates that h-BNNS can be a promising protective coating for Ag based items such as jewelry or mirrors used in astronomical telescopes.

\section{INTRODUCTION}

Two-dimensional (2D) materials with thickness less than a few nanometers have recently been investigated as a new class of protective coatings. 2D materials such as graphene and h-BNNS are among the most studied ones, since they have many desirable properties such as high mechanical strength and flexibility [1,2], high thermal conductivity [3], impermeability to water and gases [4], and high optical transparency [5]. Most importantly, being atomically thin, these 2D materials will not affect applications that require high levels of dimension precision.

h-BNNS and graphene, however, also have different physical and chemical properties, with the former being highly stable in air at elevated temperatures and electrically insulating. Due to high electrical conductivity, graphene actually contributes to the formation of galvanic cells with the underlying metal substrates, thus accelerating the corrosion process over long terms [6]. In comparison, h-BNNS also has excellent chemical stability in various aquatic environments [7]. Furthermore, hexagonal boron nitride nanomaterials are non-cytotoxic and have higher levels of biocompatibility when compared to their carbon counterparts [8-11]. In a recent study, a $130 \mu \mathrm{m}$ thick h-BN coating was used as a protection layer of a solar probe owing to h-BN's stability at high temperatures and low ratios of the solar absorptivity to the emissivity [12]. All these properties render h-BNNS a desirable protective coating that can be used in harsh environments. 
Due to its high reflectivity in the UV-visible range, Ag mirrors are believed to be a great choice in astronomical telescopes in earth-based observatories [13]. However, Ag slowly tarnishes in air as the consequence of reaction with sulfur containing compounds especially $\mathrm{H}_{2} \mathrm{~S}$, producing black $\mathrm{Ag}_{2} \mathrm{~S}$ on the surface resulting in reduced reflectivity, and added electrical resistance [14]. Methods such as polishing and soaking in a solution of sodium bicarbonate will remove the $\mathrm{Ag}_{2} \mathrm{~S}$, but can also cause irreversible material loss and create micro-pits, which will eventually lead to further corrosion. Tarnish inhibitors such as chromate conversion coatings are effective, but the process usually involves highly toxic hexavalent chromium compounds [15]. Atomic layer deposition of common barrier layers such as $\mathrm{AlO}_{\mathrm{x}}$ is also incompatible with $\mathrm{Ag}$ because this process can cause partial oxidation on Ag surface, which will compromise the reflectivity [13]. Therefore, the research on ultra-thin protective coatings with non-toxic materials that are compatible with Ag as corrosion inhibitors is of great importance.

Studies have been carried out on CVD synthesis of h-BNNS on certain facets of single crystal Ag [16-18], but the h-BNNS growth on widely available polycrystalline Ag foil via a relatively simple atmospheric pressure (AP)-CVD process has been rarely reported. While most anticorrosion studies utilizing 2D materials were conducted on copper, nickel, and stainless steel [1923], the anti-corrosion behavior of h-BNNS thin film on commercial Ag foil has not been studied before. In this work, we show that high-quality h-BNNS can be grown on polycrystalline Ag foils via AP-CVD, without affecting the optical properties of the Ag surface. We also show that the protected $\mathrm{Ag}$ shows enhanced anti-corrosion properties against $\mathrm{H}_{2} \mathrm{~S}$. 


\section{EXPERIMENTAL}

Growth of h-BNNS thin films by CVD on Ag

The h-BNNS thin films were grown on Ag foil (0.1 mm thick, 99.9\% purity, Sigma-Aldrich) by $\mathrm{AP}-\mathrm{CVD}$ in a horizontal tube furnace. The growth temperatures were set to $940^{\circ} \mathrm{C}$ for solid $\mathrm{Ag}$ and $970^{\circ} \mathrm{C}$ for melted $\mathrm{Ag}$. Prior to the growth, $\mathrm{Ag}$ foils were annealed under $5 \%$ hydrogen in argon at $940^{\circ} \mathrm{C}$ for 5 hours to remove volatile impurities. During each growth, $100 \mathrm{mg}$ of ammonia borane $\left(98 \%\right.$, United Boron) was heated to $120^{\circ} \mathrm{C}$ to generate borazine gas. The substrates were then exposed to borazine for 20 minutes at the growth temperatures. For the growth on melted Ag, cobalt foil (0.1 mm thick, 99.95\% purity, Sigma-Aldrich) was employed as the supporting layer due to its superior wettability towards Ag.

Transferring h-BNNS thin films to TEM grids

In this work, a polymethyl methacrylate (PMMA) assisted transfer method was employed. Typically, a layer of PMMA was coated on h-BNNS/Ag by a spin coater before it was submerged in $1 \mathrm{M}$ iron(III) nitrate aqueous solution overnight. After etching, the PMMA/h-BNNS floated on the solution and was washed with deionized water several times. After that, the PMMA/h-BNNS was transferred to a TEM grid. Finally, the PMMA was removed by soaking in acetone.

Anti-corrosion tests in $\mathrm{H}_{2} \mathrm{~S}$ 
$\mathrm{H}_{2} \mathrm{~S}$ gas was generated by dropwise addition of $6 \mathrm{M} \mathrm{HCl}$ onto sodium sulfide, and delivered to a vessel containing one h-BNNS-coated $\mathrm{Ag}$ and one bare $\mathrm{Ag}$ foil. The output stream of the reactor was connected to a gas bubbler containing $\sim 50 \mathrm{~mL}$ of saturated aqueous sodium carbonate. Prior to, and after $\mathrm{H}_{2} \mathrm{~S}$ generation, the system was purged with argon to remove air or excess $\mathrm{H}_{2} \mathrm{~S}$. The total exposure time was two hours.

\section{Characterization}

Scanning electron microscopy (SEM) was conducted on JEOL-7500 equipped with a field emission gun. The SEM was operated at $1 \mathrm{kV}$ for imaging to minimize the depth of beam specimen interaction. X-ray photoelectron spectroscopy (XPS) was carried out directly on the silver foil using a PHOIBOS 100 hemispherical analyzer with an Al Ka source. Transmission electron microscopy (TEM)/scanning transmission electron microscopy (STEM) was performed on JEOL ARM 200F with an accelerating voltage of $80 \mathrm{kV}$. Diffuse reflectance spectra were acquired using a custom setup with detailed experimental information in the Supporting Information.

\section{RESULTS AND DISCUSSION}

CVD growth of h-BNNS on silver

In this work, h-BNNS thin film was grown on Ag substrates by AP-CVD, using ammonia borane as the precursor. The schematic of h-BNNS growth is shown in Figure 1. Solid and melted Ag substrates were employed as the substrates, respectively. For the growth on melted Ag substrate, 
the wettability between the melted substrate and underlying metal support is crucial. In this case, cobalt is chosen as the underlying metal support due to its superior wettability against melted Ag. Moreover, Co and Ag are almost insoluble in each other in both solid and liquid state according to the phase diagrams $[24,25]$. Therefore, the underlying Co support and the melted Ag will not form alloys which would otherwise introduce a variant to the h-BNNS growth.

Figure $2 \mathrm{a}$ and $\mathrm{b}$ show the SEM images of h-BNNS grown on solid and melted Ag substrates, respectively. The dark regions in the SEM images are the underlying Ag substrate, while h-BNNS shows different shades of grey. Since h-BNNS is insulating, the thicker film appears brighter due to charging while the thinner section looks slightly darker. More SEM images are provided in the Supporting Information (Figure S2). Comparing with h-BNNS grown on solid Ag, h-BNNS on melted Ag appears to be more uniform and contains less defects such as cracks and holes. This is because melting effectively removes surface discontinuities such as grain boundaries and surface defects, which act as the nucleation sites during early stage of the growth [26-28]. In addition, melting would create a better homogeneity in the Ag surface leading to uniform nucleation, in contrast to the localized nucleation around grain boundaries and surface defects in solid Ag. All these contribute to a growth of h-BNNS film with less defects.

XPS (Figure 2c-e) was employed to investigate the chemical composition and stoichiometry between $\mathrm{B}$ and $\mathrm{N}$ atoms. For h-BNNS films grown on solid and melted Ag, B 1s peak situated at $190.8 \mathrm{eV}$ and $\mathrm{N}$ 1s peak at $398.0 \mathrm{eV}$ are observed for both samples, which are in good agreement with the published results [29-31]. The ratio between $\mathrm{B}$ and $\mathrm{N}$ atoms calculated from the spectra is close to 1 , indicative of $1: 1$ stoichiometry in $s p^{2}$ hybridized BN. 
The selected area electron diffraction (SAED) pattern (Figure 2f) from a small region with a diameter of $100 \mathrm{~nm}$ suggests that the h-BNNS has a polycrystalline nature. A high-resolution Bright Field STEM image at the edge (Figure 2g) reveals that h-BNNS grown on melted Ag substrates is of few-atomic-layer thickness. The interlayer distance measured is $0.34 \mathrm{~nm}$ which is consistent with h-BN's (0002) spacing. The Fast Fourier Transform (FFT) image in the inset containing several sets of dots also verifies that h-BNNS is few-atomic-layer [2, 29]. High angle angular dark field (HAADF) imaging is a powerful technique that can differentiate individual atoms according to their $\mathrm{Z}$ contrast [32], with the heavier the atom the higher intensity in the HAADF image. STEM-HAADF image of a monolayer region (Figure S1d) clearly shows the honeycomb structure of alternating $\mathrm{B}$ and $\mathrm{N}$ atoms, with $\mathrm{B}$ atoms associated with the darker spots and $\mathrm{N}$ atoms with the brighter ones. Furthermore, electron energy loss spectroscopy (EELS) was employed to determine the chemical state of B and N. The EELS spectrum (Figure $2 \mathrm{~h}$ ) shows two bands starting at 188 and $398 \mathrm{eV}$, corresponding respectively to K-shell ionization edge of B and $\mathrm{N}$. Each band shows well-resolved $1 \mathrm{~s}-\pi^{*}$ and $1 \mathrm{~s}-\sigma^{*}$ antibonding orbits indicating a $s p^{2}$ hybridized h-BN structure [33]. Highly crystalline h-BN can generate a sharp Raman band at $\sim 1366 \mathrm{~cm}^{-1}$, but its intensity is much lower than its carbon counterpart [34]. Due to the low crystallinity and fewatomic-layer thickness, h-BN characteristic Raman G band was not observed using a $632.81 \mathrm{~nm}$ laser source.

There is invisible difference in appearance between the h-BNNS grown on solid Ag (denoted hBNNS_Ag) and bare annealed Ag (Bare_Ag). Diffused reflectance spectra were collected to assess if the h-BNNS protective coating alters the optical property of Ag. The spectra (Figure 3) 
demonstrate that the h-BNNS_Ag and Bare_Ag show only minor difference $(\sim 2.5 \%)$ in the reflectance in the UV-visible light range. The h-BNNS-coated Ag reflects UV-Visible light effectively due to a couple of reasons. Firstly, h-BN itself has a large bandgap of around $5.5 \mathrm{eV}$, which means the coating does not absorb light with wavelength longer than $215 \mathrm{~nm}$ [35]. Secondly, the h-BNNS is only few-atomic-layer thick and therefore has a high level of transparency to the light. Furthermore, $\mathrm{h}-\mathrm{BN}$ is atomically flat where the hexagons are aligned in a nearly perfect atomic plane, which does not negatively interfere with reflection. Note that the reflectance is relatively low compared to Ag films or coatings purposely prepared for mirrors $[13,36]$. This is because that the Ag foils used in this research were produced via metal rolling which leaves many surface defects such as strips and pinholes, affecting the reflectivity. Therefore, it is reasonable to say that h-BNNS coatings would not affect the application of $\mathrm{Ag}$ as mirrors.

Anti-corrosion performance

Ag is susceptible to tarnishing due to sulfide formation even in natural conditions where $\mathrm{H}_{2} \mathrm{~S}$ concentration is very low $(0.001-0.005 \mathrm{ppm})[37,38]$. To assess the efficacy of the h-BNNS protective film, corrosion tests were conducted in a flask filled with $\mathrm{H}_{2} \mathrm{~S}$ gas in damp environment. Despite the quality of h-BNNS grown on melted Ag substrate is better, from a practical point of view, h-BNNS grown on solid Ag was used for the anti-corrosion tests. On solid Ag, h-BNNS tends to have more defects such as cracks and holes (Figure 2a and 2b).

The h-BNNS_Ag and Bare_Ag were placed next to each other in a flask filled with $\mathrm{H}_{2} \mathrm{~S}$. After 30-minute exposure, the bare Ag foil (denoted Bare_Ag_ $\mathrm{H}_{2} \mathrm{~S}$ ) showed highly visible 
decolorization. The initial shiny surface with metallic glare gradually darkened. After two hours of exposure, the surface of bare Ag foil showed a brownish color. In comparison, the surface color and metallic luster of the coated foil (denoted h-BNNS_Ag_ $\mathrm{H}_{2} \mathrm{~S}$ ) almost remained the same. Optical images (Figure 4) collected are in line with the visual observation. Noticeably, the color of certain crystal grains is darker than others in the Bare_Ag_ $\mathrm{H}_{2} \mathrm{~S}$ sample. This indicates that certain exposed facets of $\mathrm{Ag}$ are more reactive towards $\mathrm{H}_{2} \mathrm{~S}$ [39]. In comparison, the uniformity of color of the h-BNNS_Ag_ $\mathrm{H}_{2} \mathrm{~S}$ is indicative of minimal tarnishing. Moreover, based upon SEM studies (Figure S4), the surface of Bare_Ag appears to be textured due to formation of nanoparticles after $\mathrm{H}_{2} \mathrm{~S}$ exposure, while the surface morphology of h-BNNS_Ag_ $\mathrm{H}_{2} \mathrm{~S}$ remains largely unchanged.

Diffuse reflectance spectra (Figure 5) were collected to evaluate the impact of $\mathrm{H}_{2} \mathrm{~S}$ exposure on the reflectivity of Ag. The Bare_Ag_H $\mathrm{H}_{2} \mathrm{~S}$ sample shows a significant decrease in reflectance in the visible range after the exposure. On the contrary, h-BNNS_Ag_ $\mathrm{H}_{2} \mathrm{~S}$ shows only a minor reflectivity decrease with respect to samples without exposure, indicating that BNNS thin films effectively protects Ag substrate. The attenuation in reflectance of h-BNNS_Ag and Bare_Ag in Figure 3 appears to be slightly lower than those shown in Figure 5. This is likely due to the different spots (circles with $1 \mathrm{~mm}$ radius) selected for acquiring the spectra. The commercial $\mathrm{Ag}$ foils used in this research were not polished. Therefore, the surface defects such as scratches and pinholes can affect the reflectivity of samples. XPS spectra of Bare_Ag_ $\mathrm{H}_{2} \mathrm{~S}$ and h-BNNS_Ag_H $\mathrm{H}_{2} \mathrm{~S}$ (Figure 6) reveal the intensity difference of $S 2 p$ and $2 s$ peaks. The atomic ratio of $S$ to $A g$ can be calculated from the spectra. The Bare_Ag_ $\mathrm{H}_{2} \mathrm{~S}$ has a $\mathrm{S}$ to $\mathrm{Ag}$ ratio of 0.215 which is 5 times higher than that of the h-BNNS_Ag_H $\mathrm{H}_{2} \mathrm{~S}$ sample. The Ag XPS spectra consist of two peaks, $\mathrm{Ag} 3 \mathrm{~d}_{5 / 2}$ and $\mathrm{Ag} 3 \mathrm{~d}_{3 / 2}$, 
among which $\mathrm{Ag} 3 \mathrm{~d}_{5 / 2}$ is often used when discussing the formation of $\mathrm{Ag}^{+}[37,40,41]$. Based upon deconvolution, $\mathrm{Ag} 3 \mathrm{~d}_{3 / 2}$ shows no significant difference in the components, whereas, for the $\mathrm{Ag}$ $3 \mathrm{~d}_{5 / 2}$ peak, a remarkably larger component situated at $377.7 \mathrm{eV}, 0.4 \mathrm{eV}$ lower than the $\mathrm{Ag}$ metallic peak, is found for the Bare_Ag_H $\mathrm{H}_{2} \mathrm{~S}$, attributing to a large quantity of $\mathrm{Ag}_{2} \mathrm{~S}$ formed on the surface $[37,42]$.

As noted above, h-BNNS films grown on solid Ag tends to have more cracks and holes, which expose $\mathrm{Ag}$ to $\mathrm{H}_{2} \mathrm{~S}$ during the tests. The quality of h-BNNS films is affected by the physical features of $\mathrm{Ag}$ surface including grain boundaries and defects, which in turn determined by how the $\mathrm{Ag}$ items are produced, such as rolling, casting, and physical vapor deposition. Commercial Ag mirrors produced by deposition are relatively free of defects and are expected to lead to high quality h-BNNS films, which are expected to exert better protection on the underlying mirrors. Transferring h-BNNS to commercial Ag items can protect them from tarnishing to some degree. However, transferring CVD films is a laborious task. The quality of the transferred films often suffers due to contamination from the etching solution and damage caused by mechanical forces exerted on the films. Using h-BNNS directly grown on the substrate for protection is a relatively simple and practical process.

\section{CONCLUSION}

In this work, atomically thin h-BNNS was successfully grown on solid and melted Ag substrates, respectively, via AP-CVD using ammonia borane as a precursor. The h-BNNS grown on melted 
$\mathrm{Ag}$ appears to be continous and uniform in thickness because the melting process effectivley removes highly sporadic defects and impurities that act as nucleation sites. The ultra-thin h-BNNS on solid Ag substrate poses negligable impact on metallic luster and appearance. In accelerated corrosion tests, the Ag substrate coated by h-BNNS showed invisible optical color change under high concentration of $\mathrm{H}_{2} \mathrm{~S}$, while bare Ag displayed drastic color change within two hours of exposure. XPS analysis reveals that sulfide formation was largely suppressed for the coated Ag substrate, which means the h-BNNS coating effectively impedes Ag tarnishing in an environment with high level of $\mathrm{H}_{2} \mathrm{~S}$. These results suggest that h-BNNS can be grown on Ag substrate and it is a good candidate as an ultra-thin protective coating against $\mathrm{Ag}$ tarnishing. It is expected that the high chemical stability and thermal stability of h-BNNS will also make it an effective coating for $\mathrm{Ag}$ mirrors that are used at elevated temperatures and other corrosive environments. 


\section{FIGURES}

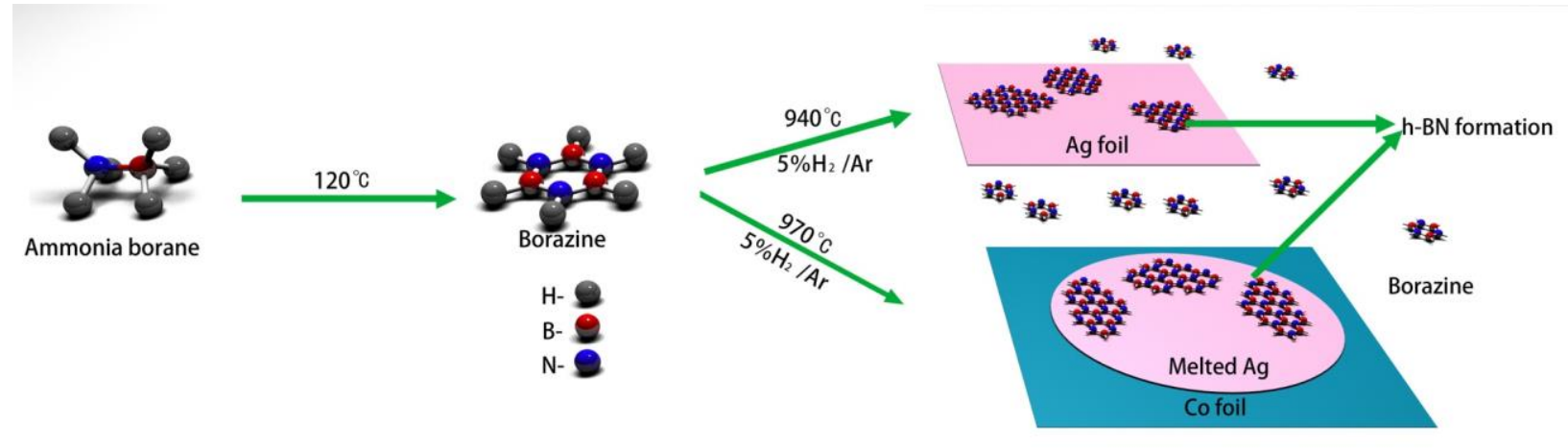

Figure 1. Schematic of h-BNNS growth on both solid and melted Ag substrates. 

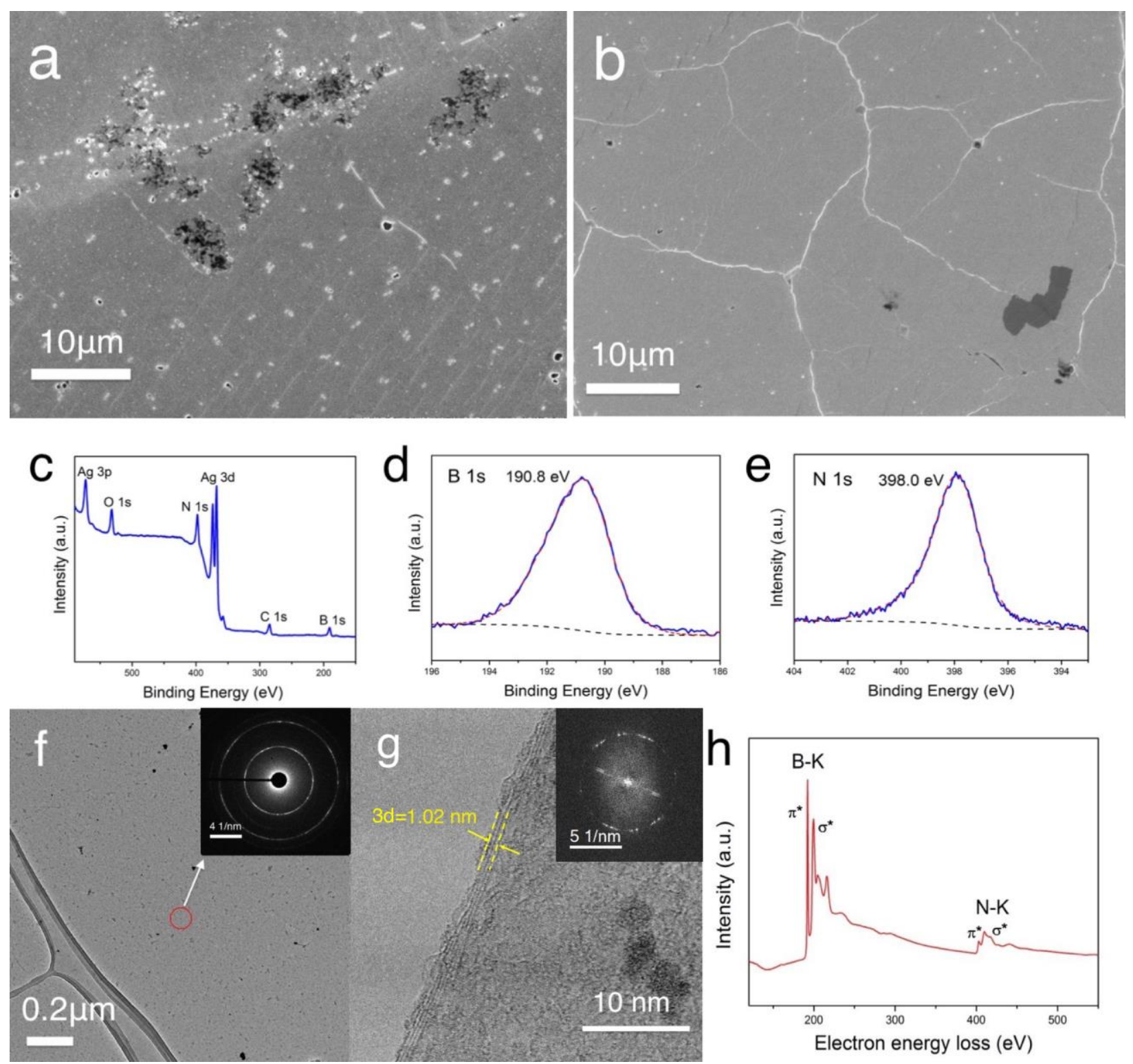

Figure 2. Characterization of h-BNNS grown on Ag substrates. (a) and (b) SEM images of hBNNS grown on solid and melted Ag substrates, respectively. (c), (d) and (e) XPS survey, B 1s and $\mathrm{N}$ 1s spectra of h-BNNS. (f) TEM image and SAED pattern (inset) of a region with a diameter of $100 \mathrm{~nm}$ on h-BNNS. (g) High resolution Bright Field STEM image and the FFT pattern (inset) of h-BNNS. (h) EELS spectrum. 


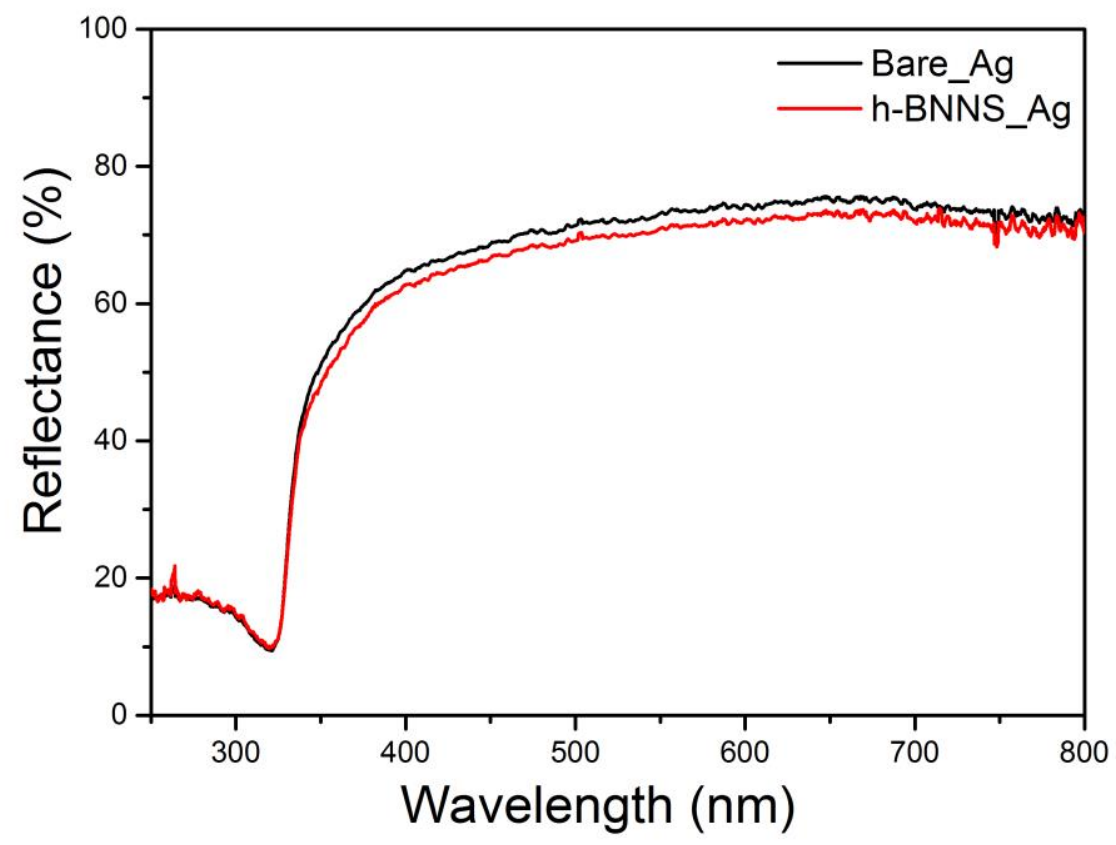

Figure 3. UV-Vis diffuse reflectance spectra of annealed Ag (Bare_Ag) and h-BNNS-coated Ag (h-BNNS_Ag). 

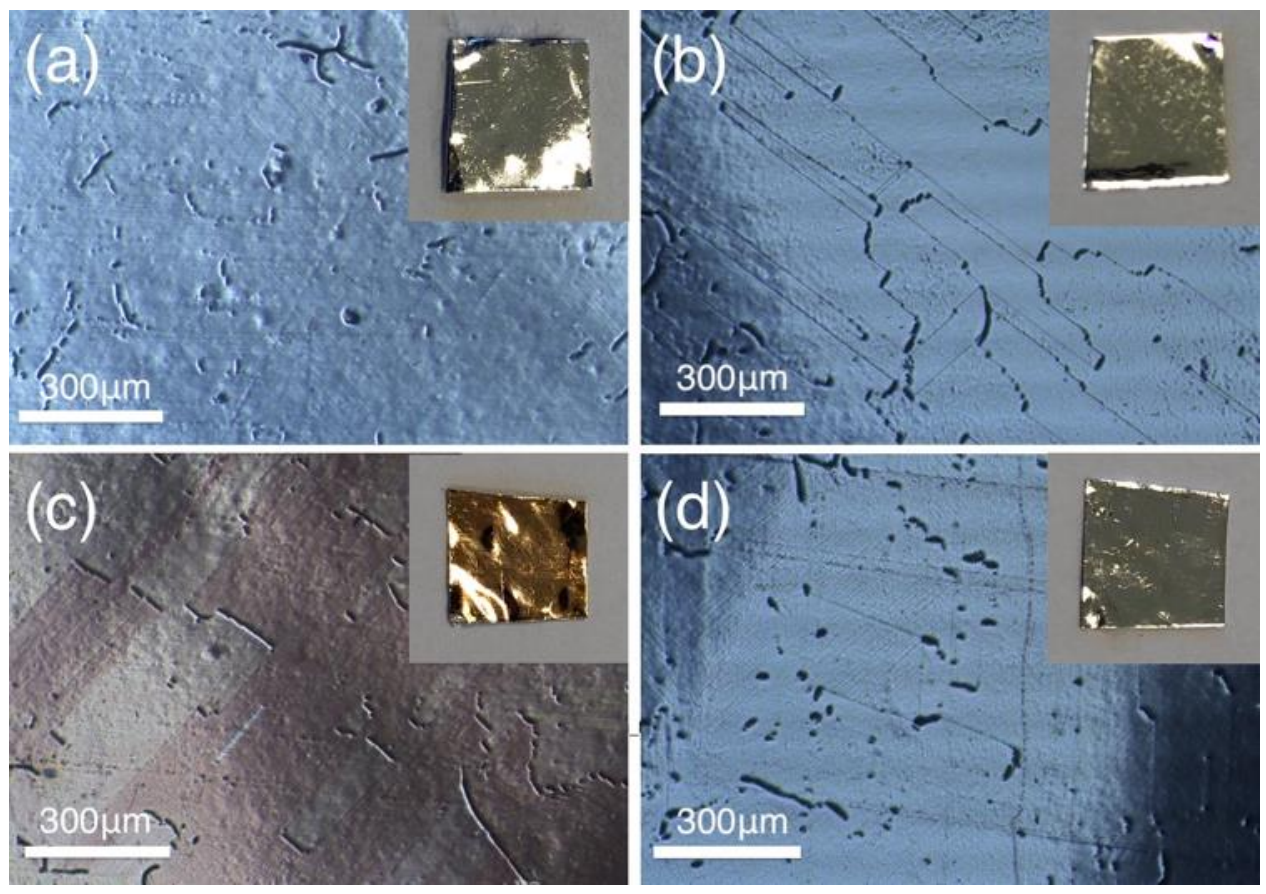

Figure 4. Optical images of (a) Bare_Ag; (b) h-BNNS_Ag; (c) Bare_Ag_H $\mathrm{H}_{2} \mathrm{~S}$; (d) hBNNS_Ag_ $\mathrm{H}_{2} \mathrm{~S}$. Insets are photos taken by a digital camera. 


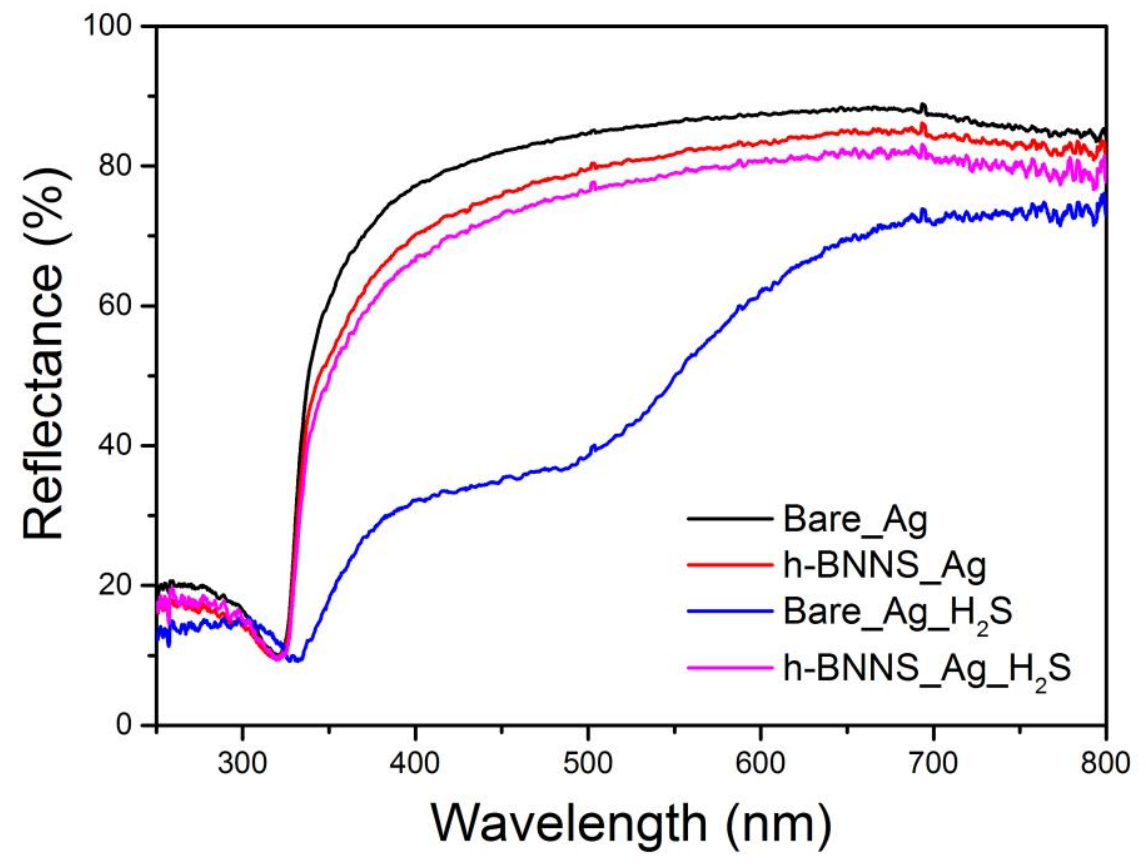

Figure 5. UV-Vis diffuse reflectance spectra for Bare_Ag, h-BNNS_Ag, Bare_Ag_H $\mathrm{H}_{2} \mathrm{~S}$ and $\mathrm{h}$ BNNS_Ag_ $\mathrm{H}_{2} \mathrm{~S}$. 

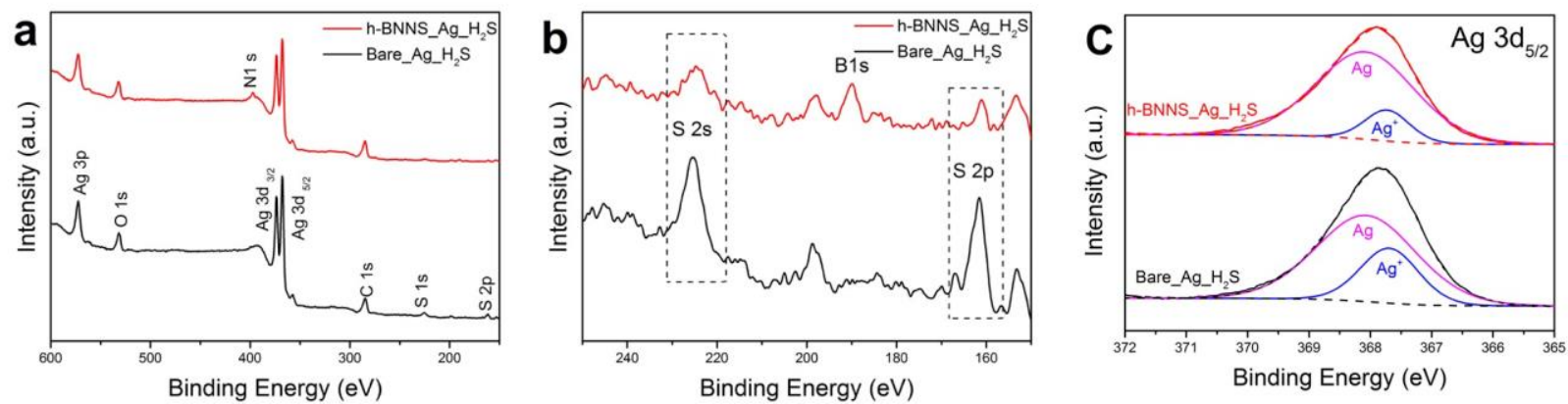

Figure 6. XPS spectra of Bare_Ag_H $\mathrm{H}_{2} \mathrm{~S}$ and h-BNNS_Ag_H ${ }_{2} \mathrm{~S}$. (a) survey spectra; (b) S 2s and S $2 p$ peaks; (c) $\mathrm{Ag} 3 \mathrm{~d}_{5 / 2}$ peaks with metallic $\mathrm{Ag}$ and $\mathrm{Ag}^{+}$components. 


\section{ASSOCIATED CONTENT}

Supporting information 1: UV-Vis custom setup details, Atomic resolution TEM images, SEM constrat explanation, digital photos of corrosion experiments, SEM images showing fine morphological change after $\mathrm{H}_{2} \mathrm{~S}$ exposure and Ag XPS spectra. (docx)

\section{AUTHOR INFORMATION}

\section{Corresponding Author}

Zhenguo Huang*_zhenguo.huang@uts.edu.au

Xudong Sun* $\quad$ xdsun@neu.edu.cn

\section{ACKNOWLEDGMENT}

Rui Han gratefully acknowleges China Scholarship Council (CSC) for the scholarship. The electron microscopy studies were carried out using equipments funded by an Australian Research Council (ARC) Linkage, Infrastructure, Equipment, and Facilities (LIEF) grant (LE120100104). 


\section{REFERENCES}

1. Lee, C.; Wei, X.; Kysar, J.W.; Hone, J. Measurement of the Elastic Properties and Intrinsic Strength of Monolayer Graphene. Science, 2008, 321, 385-388.

2. Kim, S.M.; Hsu, A.; Park, M.H.; Chae, S.H.; Yun, S.J.; Lee, J.S.; Cho, D.-H.; Fang, W.;

Lee, C.; Palacios, T.; Dresselhaus, M.; Kim, K.K.; Lee, Y.H.; Kong, J. Synthesis of Large-Area Multilayer Hexagonal Boron Nitride for High Material Performance. Nat. Commun., 2015, 6, 8662.

3. Jo, I.; Pettes, M.T.; Kim, J.; Watanabe, K.; Taniguchi, T.; Yao, Z.; Shi, L. Thermal Conductivity and Phonon Transport in Suspended Few-Layer Hexagonal Boron Nitride. Nano Lett., 2013, 13, 550-554.

4. Bunch, J.S.; Verbridge, S.S.; Alden, J.S.; van der Zande, A.M.; Parpia, J.M.; Craighead, H.G.; McEuen, P.L. Impermeable Atomic Membranes from Graphene Sheets. Nano Lett., 2008, $8,2458-2462$.

5. Nair, R.R.; Blake, P.; Grigorenko, A.N.; Novoselov, K.S.; Booth, T.J.; Stauber, T.; Peres, N.M.R.; Geim, A.K. Fine Structure Constant Defines Visual Transparency of Graphene. Science, 2008, 320, 1308-1308.

6. Zhou, F.; Li, Z.; Shenoy, G.J.; Li, L.; Liu, H. Enhanced Room-Temperature Corrosion of Copper in the Presence of Graphene. ACS Nano, 2013, 7, 6939-6947.

7. Pakdel, A.; Bando, Y.; Golberg, D. Nano Boron Nitride Flatland. Chem. Soc. Rev., 2014, 43, 934-959. 
8. Chen, X.; Wu, P.; Rousseas, M.; Okawa, D.; Gartner, Z.; Zettl, A.; Bertozzi, C.R. Boron Nitride Nanotubes Are Noncytotoxic and Can Be Functionalized for Interaction with Proteins and Cells. J. Am. Chem. Soc., 2009, 131, 890-891.

9. Ciofani, G.; Danti, S.; Genchi, G.G.; Mazzolai, B.; Mattoli, V. Boron Nitride Nanotubes: Biocompatibility and Potential Spill-over in Nanomedicine. Small, 2013, 9, 1672-1685.

10. Pinto, A.M.; Gonçalves, I.C.; Magalhães, F.D. Graphene-Based Materials Biocompatibility: A Review. Colloids Surf., B, 2013, 111, 188-202.

11. Weng, Q.; Wang, X.; Wang, X.; Bando, Y.; Golberg, D. Functionalized Hexagonal Boron Nitride Nanomaterials: Emerging Properties and Applications. Chem. Soc. Rev., 2016, 45, 39894012.

12. Brodu, E.; Balat-Pichelin, M. Emissivity of Boron Nitride and Metals for the Solar Probe Plus Mission. J. Spacecr. Rockets, 2016, 53, 1119-1127.

13. Fryauf, D.M.; Phillips, A.C.; Kobayashi, N.P. Corrosion Protection of Silver-Based Telescope Mirrors Using Evaporated Anti-Oxidation Overlayers and Aluminum Oxide Films by Atomic Layer Deposition. in SPIE Nanoscience + Engineering. 2016. SPIE.

14. Gay, P.A.; Bercot, P.; Pagetti, J. The Protection of Silver against Atmospheric Attack, 2004.

15. Patrick L. Hagans, C.M.H. Chromate Conversion Coatings, in ASM Handbook, Volume 5: Surface Engineering, C.M Cotell, J.A.S., and F.A. Smidt, Jr., Editor. 1994, ASM International. pp 405-411.

16. Müller, F.; Hüfner, S.; Sachdev, H.; Laskowski, R.; Blaha, P.; Schwarz, K. Epitaxial Growth of Hexagonal Boron Nitride on Ag(111). Phys. Rev. B., 2010, 82, 113406. 
17. Müller, F.; Grandthyll, S. Monolayer Formation of Hexagonal Boron Nitride on $\operatorname{Ag}(001)$. Surf. Sci., 2013, 617, 207-210.

18. Garnica, M.; Schwarz, M.; Ducke, J.; He, Y.; Bischoff, F.; Barth, J.V.; Auwärter, W.; Stradi, D. Comparative Study of the Interfaces of Graphene and Hexagonal Boron Nitride with Silver. Phys. Rev. B., 2016, 94, 155431.

19. Li, L.H.; Xing, T.; Chen, Y.; Jones, R. Boron Nitride Nanosheets for Metal Protection. Adv. Mater. Interfaces, 2014, 1, 1300132.

20. Mahvash, F.; Eissa, S.; Bordjiba, T.; Tavares, A.C.; Szkopek, T.; Siaj, M. Corrosion Resistance of Monolayer Hexagonal Boron Nitride on Copper. Sci. Rep., 2017, 7, 42139.

21. Husain, E.; Narayanan, T.N.; Taha-Tijerina, J.J.; Vinod, S.; Vajtai, R.; Ajayan, P.M. Marine Corrosion Protective Coatings of Hexagonal Boron Nitride Thin Films on Stainless Steel. ACS Appl. Mater. Interfaces, 2013, 5, 4129-4135.

22. Liu, Z.; Gong, Y.; Zhou, W.; Ma, L.; Yu, J.; Idrobo, J.C.; Jung, J.; MacDonald, A.H.; Vajtai, R.; Lou, J.; Ajayan, P.M. Ultrathin High-Temperature Oxidation-Resistant Coatings of Hexagonal Boron Nitride. Nat. Commun., 2013, 4, 2541.

23. Khan, M.H.; Jamali, S.S.; Lyalin, A.; Molino, P.J.; Jiang, L.; Liu, H.K.; Taketsugu, T.; Huang, Z. Atomically Thin Hexagonal Boron Nitride Nanofilm for Cu Protection: The Importance of Film Perfection. Adv. Mater., 2017, 29, 1603937.

24. Hansen, M.; Anderko, K.; Salzberg, H.W. Constitution of Binary Alloys. J. Electrochem. Soc., 1958, 105, 260C-261C. 
25. Karakaya, I.; Thompson, W.T. The Ag-Co (Silver-Cobalt) System. Bull. Alloy Phase Diagrams, 1986, 7, 259-263.

26. Khan, M.H.; Huang, Z.; Xiao, F.; Casillas, G.; Chen, Z.; Molino, P.J.; Liu, H.K. Synthesis of Large and Few Atomic Layers of Hexagonal Boron Nitride on Melted Copper. Sci. Rep., 2015, $5,7743$.

27. Khan, M.H.; Liu, H.K.; Sun, X.; Yamauchi, Y.; Bando, Y.; Golberg, D.; Huang, Z. FewAtomic-Layered Hexagonal Boron Nitride: CVD Growth, Characterization, and Applications. Mater. Today, 2017, 20, 611-628.

28. Khan, M.H.; Casillas, G.; Mitchell, D.R.G.; Liu, H.K.; Jiang, L.; Huang, Z. Carbon- and Crack-Free Growth of Hexagonal Boron Nitride Nanosheets and Their Uncommon Stacking Order. Nanoscale, 2016, 8, 15926-15933.

29. Song, L.; Ci, L.; Lu, H.; Sorokin, P.B.; Jin, C.; Ni, J.; Kvashnin, A.G.; Kvashnin, D.G.; Lou, J.; Yakobson, B.I.; Ajayan, P.M. Large Scale Growth and Characterization of Atomic Hexagonal Boron Nitride Layers. Nano Lett., 2010, 10, 3209-3215.

30. Kim, K.K.; Hsu, A.; Jia, X.; Kim, S.M.; Shi, Y.; Hofmann, M.; Nezich, D.; RodriguezNieva, J.F.; Dresselhaus, M.; Palacios, T.; Kong, J. Synthesis of Monolayer Hexagonal Boron Nitride on Cu Foil Using Chemical Vapor Deposition. Nano Lett., 2012, 12, 161-166.

31. Shi, Y.; Hamsen, C.; Jia, X.; Kim, K.K.; Reina, A.; Hofmann, M.; Hsu, A.L.; Zhang, K.; Li, H.; Juang, Z.-Y.; Dresselhaus, M.S.; Li, L.-J.; Kong, J. Synthesis of Few-Layer Hexagonal Boron Nitride Thin Film by Chemical Vapor Deposition. Nano Lett., 2010, 10, 4134-4139. 
32. Krivanek, O.L.; Chisholm, M.F.; Nicolosi, V.; Pennycook, T.J.; Corbin, G.J.; Dellby, N.; Murfitt, M.F.; Own, C.S.; Szilagyi, Z.S.; Oxley, M.P.; Pantelides, S.T.; Pennycook, S.J. Atom-byAtom Structural and Chemical Analysis by Annular Dark-Field Electron Microscopy. Nature, 2010, 464, 571.

33. Chopra, N.G.; Luyken, R.J.; Cherrey, K.; Crespi, V.H.; Cohen, M.L.; Louie, S.G.; Zettl, A. Boron Nitride Nanotubes. Science, 1995, 269, 966-967.

34. Stenger, I.; Schué, L.; Boukhicha, M.; Berini, B.; Plaçais, B.; Loiseau, A.; Barjon, J. Low Frequency Raman Spectroscopy of Few-Atomic-Layer Thick h-BN Crystals. 2D Mater., 2017, 4, 031003.

35. Watanabe, K.; Taniguchi, T.; Kanda, H. Direct-Bandgap Properties and Evidence for Ultraviolet Lasing of Hexagonal Boron Nitride Single Crystal. Nat. Mater., 2004, 3, 404.

36. Hass, G.; Heaney, J.B.; Herzig, H.; Osantowski, J.F.; Triolo, J.J. Reflectance and Durability of Ag Mirrors Coated with Thin Layers of $\mathrm{Al}_{2} \mathrm{O}_{3}$ Plus Reactively Deposited Silicon Oxide. Appl. Opt., 1975, 14, 2639-2644.

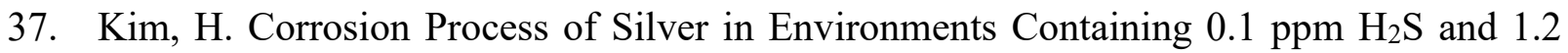
ppm $\mathrm{NO}_{2}$. Mater. Corros., 2003, 54, 243-250.

38. Pope, D.; Gibbens, H.R.; Moss, R.L. The Tarnishing of Ag at Naturally-Occurring $\mathrm{H}_{2} \mathrm{~S}$ and $\mathrm{SO}_{2}$ Levels. Corros. Sci., 1968, 8, 883-887.

39. Sundquist, B.E. A Direct Determination of the Anisotropy of the Surface Free Energy of Solid Gold, Silver, Copper, Nickel, and Alpha and Gamma Iron. Acta Metall., 1964, 12, 67-86. 
40. Krylova, V.; Dukštienè, N. Synthesis and Characterization of Ag2s Layers Formed on Polypropylene. Journal of Chemistry, 2013, 2013, 11.

41. Shukla, S.; Seal, S.; Mishra, S.R. Synthesis and Characterization of Silver Sulfide Nanoparticles Containing Sol-Gel Derived Hpc-Silica Film for Ion-Selective Electrode Application. J. Sol-Gel Sci. Technol., 2002, 23, 151-164.

42. Pang, M.; Hu, J.; Zeng, H.C. Synthesis, Morphological Control, and Antibacterial Properties of Hollow/Solid $\mathrm{Ag}_{2} \mathrm{~S} / \mathrm{Ag}$ Heterodimers. J. Am. Chem. Soc., 2010, 132, 10771-10785. 
Table of Contents/Abstract Graphic

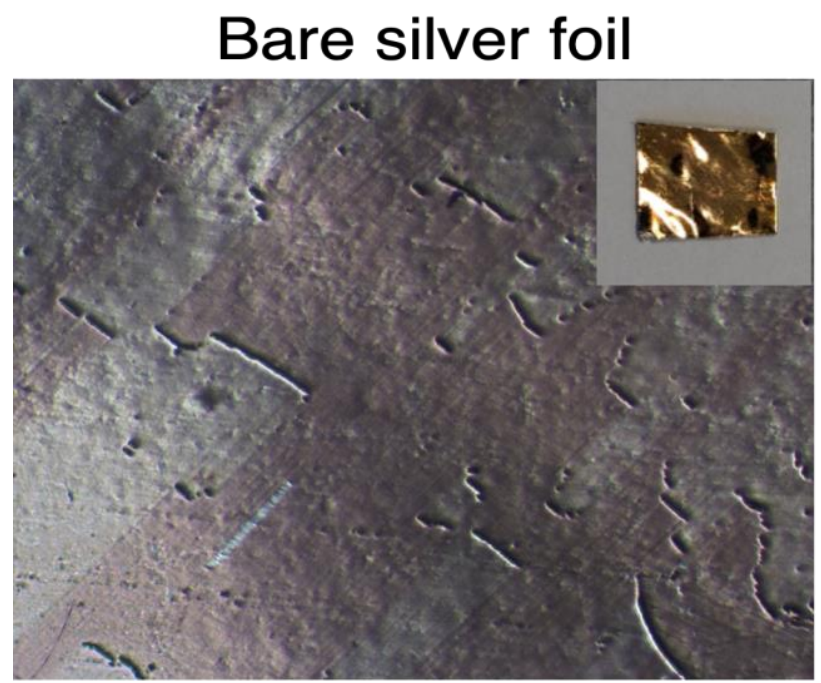

\section{h-BNNS-coated silver foil}

After 2 hours of $\mathrm{H}_{2} \mathrm{~S}$ exposure 